\title{
Buried Interfaces and Phase Transformations in a Biogenic Single Crystal
}

\author{
C.-H. Wu, ${ }^{1}$ R. Knapp, ${ }^{1}$ C. Tester, ${ }^{1}$ W. Gu, ${ }^{2}$ D. Jang, ${ }^{2}$ J. Greer, ${ }^{2}$ B. Lai, ${ }^{3}$ S. Chen, ${ }^{3}$ C. Sun, ${ }^{3}$ M \\ Balasubramanian, ${ }^{3}$ and D. Joester ${ }^{1}$ \\ ${ }^{1}$ Department of Materials Science and Engineering, Northwestern University, Evanston, IL 60202 \\ ${ }^{2}$ Materials Science and Mechanics, Caltech, Pasadena, CA 91125 \\ ${ }^{3}$ Advanced Photon Source, Argonne National Laboratory, Argonne, IL 60439
}

Biomineralized tissues are highly evolved organic-inorganic composites that provide mechanical support and are integral to sensing and homeostasis in a wide variety of organisms. ${ }^{[1]}$ An important aspect of biomineral structure and function is the organic matrix that interacts with the forming mineral and is occluded during mineralization. The analysis of the role of matrix molecules has been a considerable challenge, given the hard/soft hybrid nature of the final composite, its chemical and structural complexity, and the low atomic number of the majority of its constituents.

For example, biomineralization in the sea urchin embryo results in oriented single crystalline spicules with complex, branching shape and smoothly curving surfaces (Fig. 1A). ${ }^{[2]}$ Unlike typical bulk crystal growth from solution, however, it has been shown that PMCs precipitate at least one transient amorphous phase that converts into the final, single-crystalline biomineral. ${ }^{[3]}$ The mechanism of the disorder-to-order transformation remains poorly understood. While the weight fraction of organics occluded in the final crystal is less than $0.5 \mathrm{wt} \%$, there are more than 200 different proteins present. ${ }^{[4]}$ The role of these proteins is not known, but may involve modulating a) surface energy during crystal growth; b) phase transformation kinetics of metastable precursors; and c) mechanical properties. We recently discovered that a recombinant protein, sea urchin vascular endothelial growth factor (rVEGF), controls spicule shape in PMC culture. ${ }^{[5]}$ Depending on rVEGF concentration in the medium, PMC deposit linear, " $\mathrm{h} / \mathrm{H}$ "-shaped, or triradiate spicules. Remarkably, the change from linear to triradiate occurs with a switch from bidirectional growth parallel to the calcite $c$-axis to growth along the three $a$ axes (Fig. 1B/C).

Our long-term objective is to understand the role of individual proteins and emergent properties that result from the ensemble. Towards this goal, we are using electron optical methods (STEM-HAADF) to investigate the distribution of organics in the final biomineral. There is clear evidence for preferential adsorption of proteins on specific crystallographic faces (Fig. 1D). Patterns of preferential adsorption depend on the crystallographic growth direction, supporting the hypothesis that at least some proteins are involved in modulating surface energy to control crystal growth. At the same time, three-point bending tests of in vitro-grown single crystals indicate an important role of organics for the strength of the biomineral (Fig. 1E). In parallel to these experiments, we investigate the evolution of order in the biomineral using strontium as a pulse-chase probe for X-ray fluorescence microscopy (XFM, Fig. 2A) and X-ray absorption spectroscopy ( $\mu$-XANES and bulk XAS; Fig. 2B-D). Based on Sr K-edge XAS of cryo-frozen whole embryos, we propose that the initially deposited calcium carbonate has short-range order resembling synthetic hydrated amorphous calcium carbonate (ACC) ${ }^{[6]}$ Within $3 \mathrm{~h}$, the short-range order of calcite is established. Between $3 \mathrm{~h}$ and $24 \mathrm{~h}$, the short-range order does not change, while longrange order increases. ${ }^{[7]}$ In summary, the ability to stimulate and control the crystal deposition process in vitro sets the stage for a an in depth analysis of a complex hard/soft nanocomposite using electron and $\mathrm{X}$-ray imaging and scattering approaches. We are confident that in combination with genetic 
engineering, our model system will enable unprecedented insights into biological single crystal growth and functional properties.
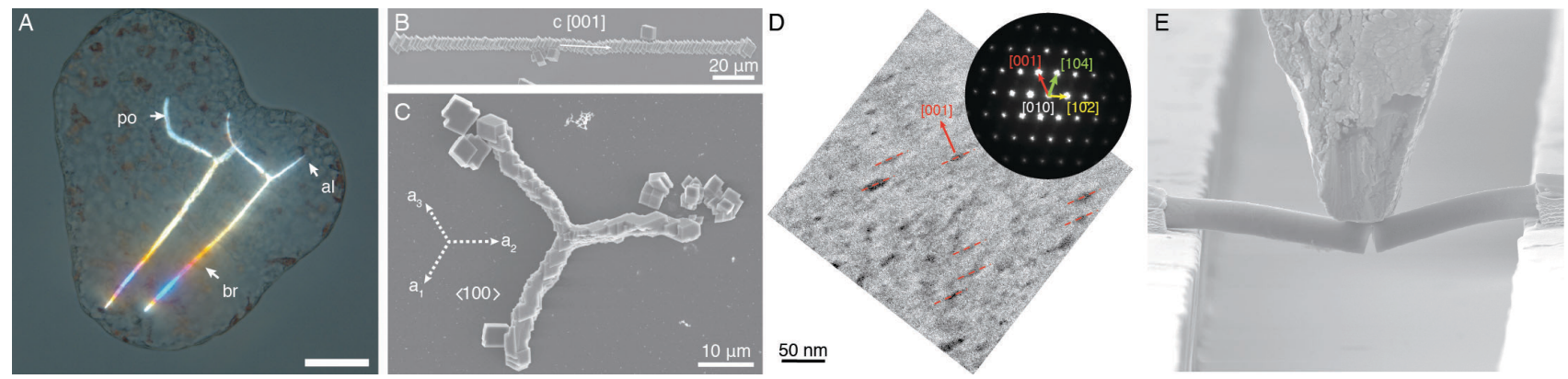

FIG. 1. A. DIC image of the endoskeletal spicules of a pluteus stage embryo of $S$. purpuratus. B-C. SEM images of linear (B) and triradiate (C) spicules deposited in vitro by PMC at low and high rVEGF concentration, respectively. After removal of soft tissues, spicules were overgrown with synthetic calcite by exposure to a supersaturated solution of calcium carbonate. D. STEM-HAADF reveals low $Z$ volumes inside a lamella parallel to (010) prepared from a triradiate spicule by FIB milling. These volumes likely correspond to protein adsorbed on the (001) plane. E. This spicule was loaded to failure in a three point bending test in the Greer Group SEMentor.
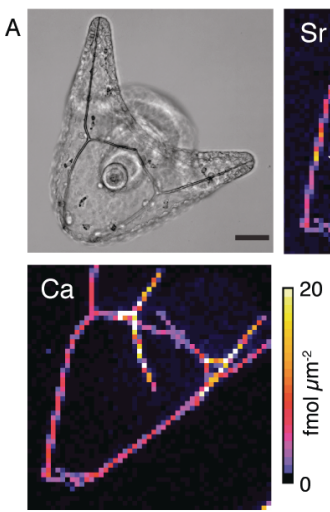

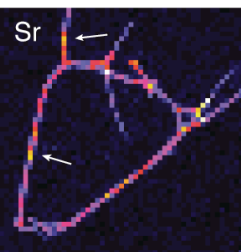

20

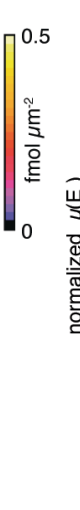

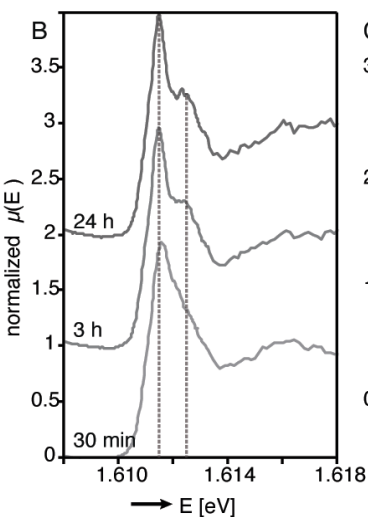


FIG. 2. A. Light microscopy image of pluteus stage L. pictus and XFM Sr and Ca maps of embryo pulse-chase labeled Sr in the growth medium. B. Sr K-edge $\mu$ XANES spectra recorded after $0.5 \mathrm{~h}, 3 \mathrm{~h}$, and $24 \mathrm{~h}$ show increasing intensity of a peak at $\mathrm{E}=16,126 \mathrm{eV}$ that is characteristic of calcite. C. Sr K-edge bulk XANES spectra are qualitatively identical to $\mu$ XANES spectra, but have significantly improved signal-to-noise ratio. D. Bulk Sr K-edge EXAFS R-space spectra demonstrate the evolution of calcite order.

\section{References:}

[1] H. A. Lowenstam, S. Weiner, in "On biomineralization", (Oxford University Press, USA).

[2] F. H. Wilt, Dev Biol 280, (2005).

[3] Y. U. T. Gong, C. E. Killian, I. C. Olson, N. P. Appathurai, A. L. Amasino, M. C. Martin, L. J. Holt, F. H. Wilt, P. Gilbert, Proc. Natl. Acad. Sci. U. S. A. 109, (2012).

[4] K. Mann, F. H. Wilt, A. J. Poustka, Proteome Sci 8, (2010).

[5] R. T. Knapp, C.-H. Wu, K. C. Mobilia, D. Joester, J Am Chem Soc 134, (2012).

[6] C. Tester, C.-H. Wu, M. R. Krejci, L. Mueller, A. Park, B. Lai, S. Chen, C. Sun, M. Balasubramanian, D. Joester, Adv Fun Mat, (in press).

[7] This work was supported in part by the US National Science Foundation (DMR-0805313 and DMR-1106208), the International Institute for Nanotechnology, and the MRSEC program of the National Science Foundation (DMR-1121262) at the Materials Research Center of Northwestern University (NU). 